\title{
Considerações sobre o Algoritmo PNLMS com Fatores de Ativação Individuais
}

\author{
Francisco das Chagas de Souza, Orlando José Tobias e Rui Seara
}

\begin{abstract}
Resumo-Neste artigo, um algoritmo LMS normalizado proporcional (PNLMS) usando fatores de ativação individuais é proposto. Em contraste com o algoritmo PNLMS padrão, que atribui um único fator de ativação para todos os coeficientes do filtro adaptativo, o algoritmo proposto atribui um fator de ativação para cada coeficiente. Dessa forma, para respostas ao impulso com elevada esparsidade, a estratégia proposta conduz a uma melhor distribuição da energia de adaptação entre os coeficientes do filtro. Resultados de simulação verificam a eficácia da abordagem proposta.
\end{abstract}

Palavras-chave-Algoritmo LMS normalizado proporcional (PNLMS), filtragem adaptativa, identificação de sistema, resposta ao impulso esparsa.

Abstract-This paper proposes a proportionate normalized least-mean-square (PNLMS) algorithm using individual activation factors. In contrast to the PNLMS algorithm, which considers a global activation factor for all adaptive filter coefficients, the proposed algorithm uses an individual activation factor for each filter coefficient. Thus, for impulse responses with high sparseness, the proposed strategy leads to a better distribution of the adaptation energy over the filter coefficients. Simulation results verify the effectiveness of the proposed approach.

Keywords-Proportionate normalized least-mean-square (PNLMS) algorithm, adaptive filtering, system identification, sparse impulse response.

\section{INTRODUÇÃO}

Respostas ao impulso esparsas são encontradas em muitas aplicações práticas, tais como comunicações, acústica e processos sísmicos e químicos, dentre outras [1], [2], [3]. Para essa classe de resposta ao impulso, algoritmos adaptativos clássicos, utilizando o mesmo passo de adaptação para todos os coeficientes do filtro, tal como o algoritmo LMS normalizado (NLMS), apresentam baixa velocidade de convergência. Para contornar tal problema, alguns algoritmos explorando a natureza esparsa da resposta ao impulso vêm sendo propostos na literatura [1]-[14]. Um desses algoritmos é o NLMS proporcional (PNLMS) [4]. Nesse algoritmo, cada coeficiente do filtro é atualizado proporcionalmente à sua magnitude, resultando em uma melhoria na velocidade de convergência.

Francisco das C. de Souza e Rui Seara, LINSE - Laboratório de Circuitos e Processamento de Sinais, Depto. de Eng. Elétrica, Universidade Federal de Santa Catarina, Florianópolis, SC, E-mails: \{fsouza, seara\}@linse.ufsc.br.

Orlando J. Tobias, LINSE/UFSC e Depto. Eng. Elétrica e Telecom., Universidade Regional de Blumenau, SC, E-mail: tobias.oj@ieee.org.

Este trabalho foi parcialmente financiado pela Coordenação de Aperfeiçoamento de Pessoal de Nível Superior (CAPES) e pelo Conselho Nacional de Desenvolvimento Científico e Tecnológico (CNPq).
Contudo, o algoritmo PNLMS tem seu desempenho deteriorado à medida que a esparsidade decresce [5], [6]. Versões melhoradas do algoritmo PNLMS objetivando lidar com respostas ao impulso que apresentam esparsidade média são o PNLMS++ [5] e o PNLMS melhorado (IPNLMS) [6]. Entretanto, esses algoritmos não exibem a mesma velocidade inicial de convergência obtida com o algoritmo PNLMS para respostas ao impulso com elevada esparsidade [7].

O desempenho do algoritmo PNLMS depende de alguns parâmetros predefinidos que controlam a proporcionalidade e a inicialização [1]. Assim, um ponto central é como estabelecer valores adequados para esses parâmetros, visto que eles afetam a velocidade de convergência do algoritmo. Tais parâmetros estão relacionados a uma variável do algoritmo, denominada fator de ativação, cuja função é prevenir que os coeficientes do filtro adaptativo "congelem” quando suas magnitudes são nulas ou muito pequenas. No algoritmo PNLMS, o fator de ativação é comum a todos os coeficientes, calculado amostra por amostra e dependente da norma infinita do vetor de coeficientes do filtro adaptativo. Essa abordagem considera, para obter o fator de ativação, uma distribuição de ganhos não atendendo completamente o conceito de proporcionalidade, o qual é o atributo desejado do algoritmo PNLMS. Portanto, neste trabalho, o algoritmo PNLMS será revisitado com o propósito de se obter uma nova estratégia para determinar o fator de ativação, visando melhorar o desempenho do algoritmo. Em contraste ao algoritmo PNLMS, a nova abordagem usada para calcular o fator de ativação apresenta as seguintes características:

i) Um fator de ativação individual para cada coeficiente do filtro adaptativo é utilizado.

ii) Cada fator de ativação individual é calculado em função da magnitude do correspondente coeficiente.

iii) $\mathrm{O}$ fator de ativação individual não depende dos parâmetros de proporcionalidade e inicialização, tendo em vista que tais parâmetros não estão mais presentes na formulação proposta.

Como conseqüência, a velocidade de convergência do algoritmo proposto melhora significativamente, visto que agora existe um fator de ativação individual para cada coeficiente. A nova versão do algoritmo é denominada PNLMS com fator de ativação individual (IAF-PNLMS).

Para respostas ao impulso com elevada esparsidade, resultados de simulação mostram que o algoritmo proposto apresenta maior velocidade de convergência bem como resposta mais rápida a perturbações na planta do que os algoritmos PNLMS e IPNLMS. 


\section{AlgORITMO PNLMS}

\section{A. Formulação do Algoritmo}

O algoritmo PNLMS é formulado pelo seguinte conjunto de equações [1], [6]:

Atualização dos coeficientes $(N \times 1)$

$$
\mathbf{w}(n+1)=\mathbf{w}(n)+\frac{\mu \mathbf{G}(n) e(n) \mathbf{x}(n)}{\mathbf{x}^{\mathrm{T}}(n) \mathbf{G}(n) \mathbf{x}(n)+\varepsilon}
$$

Sinal de erro

$$
e(n)=d(n)-\mathbf{w}^{\mathrm{T}}(n) \mathbf{x}(n)+z(n)
$$

Matriz de distribuição de ganhos $(N \times N)$

$$
\mathbf{G}(n)=\operatorname{diag}\left[g_{1}(\mathrm{n}) g_{2}(\mathrm{n}) \cdots g_{N}(\mathrm{n})\right]
$$

Ganho individual

$$
g_{i}(n)=\frac{\phi_{i}(n)}{\sum_{i=1}^{N} \phi_{i}(n)}
$$

Função de proporcionalidade

$$
\phi_{i}(n)=\max \left[f(n),\left|w_{i}(n)\right|\right]
$$

Fator de ativação

$$
f(n)=\rho \max \left[\delta,\|\mathbf{w}(n)\|_{\infty}\right]
$$

onde $0<\mu<2$ é o parâmetro de passo, $\varepsilon>0$ é um parâmetro de regularização (prevenindo divisão por zero e estabilizando a solução) e $\|\cdot\|_{\infty}$ caracteriza a norma infinita de um vetor. A variável $d(n)$ representa o sinal desejado e $z(n)$, um ruído de medição i.i.d. com média zero, variância $\sigma_{z}^{2}$, e não correlacionado com qualquer outro sinal do sistema. O vetor de entrada é $\mathbf{x}(n)=[x(n) x(n-1) \cdots x(n-N+1)]^{\mathrm{T}}$ e o vetor contendo os $N$ coeficientes do filtro adaptativo é dado por $\mathbf{w}(n)=\left[w_{1}(n) w_{2}(n) \cdots w_{N}(n)\right]^{\mathrm{T}}$. A matriz $\mathbf{G}(n)$ distribui os ganhos $g_{i}(n)$ entre os coeficientes, dessa forma, governando o ajuste dos passos de adaptação. O fator de ativação $f(n)$ dado em (6) depende do vetor de coeficientes do filtro adaptativo bem como de $\rho$ e $\delta$, os quais são os parâmetros de proporcionalidade (ou ativação) e inicialização, respectivamente. O parâmetro de inicialização permite a partida do processo de adaptação para $n=0$, quando todos os coeficientes do filtro são inicializados em zero. O parâmetro de proporcionalidade evita que um coeficiente individual “congele” quando sua magnitude é nula ou muito pequena [5], [6].

\section{B. Discussão e Desempenho do Algoritmo}

Nesta seção, o comportamento do algoritmo é discutido e analisado, visando obter-se uma versão melhorada do algoritmo PNLMS. Para tal, o ponto central é estudar o efeito do fator de ativação $f(n)$ e de seus parâmetros associados (proporcionalidade e inicialização) sobre o comportamento do algoritmo.
A partir de (4) e (5), $g_{i}(n)$ é reescrito como

$$
g_{i}(n)=\frac{1}{c(n)} \max \left[f(n),\left|w_{i}(n)\right|\right]
$$

com

$$
c(n)=\sum_{i=1}^{N} \phi_{i}(n)
$$

comum para todos os ganhos definidos em (7). Agora, analisando (7), algumas definições importantes são estabelecidas:

i) Ganho dos coeficientes inativos $g^{\text {inativo }}(n)$. Se $f(n)>\left|w_{i}(n)\right|$, o i-ésimo coeficiente $w_{i}(n)$ é inativo e seu ganho associado, obtido a partir de (7), é dado por

$$
g^{\text {inativo }}(n)=\frac{1}{c(n)} f(n) .
$$

Visto que o fator de ativação $f(n)$ é comum a todos os coeficientes do filtro, um ganho mínimo e comum $g^{\text {inativo }}(n)$ é atribuído a todos os coeficientes inativos. Essa é uma característica indesejável, como será mostrado mais adiante.

ii) Ganho dos coeficientes ativos $g_{i}^{\text {ativo }}(n)$. Se $f(n) \leq\left|w_{i}(n)\right|$, o $i$-ésimo coeficiente $w_{i}(n)$ é ativo e seu ganho associado, obtido a partir de (7), é dado por

$$
g_{i}^{\text {ativo }}(n)=\frac{1}{c(n)}\left|w_{i}(n)\right| \text {. }
$$

Aqui, cada ganho $g_{i}^{\text {ativo }}(n)$ está associado com a magnitude do correspondente coeficiente ativo $w_{i}(n)$. Esse ponto é a essência do algoritmo PNLMS.

Agora, considerando as definições (i) e (ii) acima, analisa-se o ganho total distribuído entre os coeficientes do filtro em cada iteração. Tal ganho pode ser representado pelo traço da matriz $\mathbf{G}(n)$. Assim,

$$
\operatorname{tr}[\mathbf{G}(n)]=\frac{N-N_{\text {ativo }}}{c(n)} f(n)+\sum_{i \in A} g_{i}(n)
$$

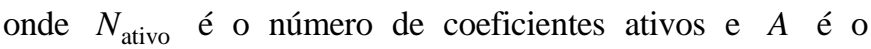
conjunto de índices associados às suas posições. Em (11), o primeiro termo do lado direito representa o ganho total distribuído entre os coeficientes inativos e o segundo, o ganho total distribuído entre os coeficientes ativos. Note que um decréscimo em $f(n)$, por exemplo, implica um aumento no segundo termo do lado direito de (11), visto que $\operatorname{tr}[\mathbf{G}(n)]$ é sempre constante e igual a 1 por construção, obtido a partir de (3) e (4) [6]. Isso significa que os ganhos associados aos coeficientes ativos também são dependentes de $f(n)$. Portanto, conclui-se que o fator de ativação $f(n)$ afeta os ganhos atribuídos aos coeficientes ativos bem como aos 
inativos. Logo, $f(n)$ tem um papel fundamental na distribuição de ganhos do algoritmo.

Neste ponto, o desempenho do algoritmo PNLMS em relação ao fator de ativação é estudado em detalhes. Para tal, simulações de Monte Carlo (MC) são obtidas (média de 100 realizações independentes) considerando um problema de identificação de sistema. O cenário para todas as simulações consiste de uma resposta ao impulso esparsa com $N=100$ coeficientes, dada em [2], com os valores de seus coeficientes ativos iguais a $\{0,1,1,0,-0,5,0,1\}$ localizados nas posições $\{1,30,35,85\}$, respectivamente. Para quantificar a esparsidade da resposta ao impulso, uma medida baseada na relação entre a norma-1 e a norma-2 da resposta ao impulso é considerada. Tal medida é definida como [3], [15]

$$
S(\mathbf{p}) \triangleq \frac{N}{N-\sqrt{N}}\left(1-\frac{\|\mathbf{p}\|_{1}}{\sqrt{N}\|\mathbf{p}\|_{2}}\right)
$$

onde o vetor $\mathbf{p}=\left[\begin{array}{llll}p_{1} & p_{2} & \cdots & p_{N}\end{array}\right]^{\mathrm{T}}$ representa a resposta ao impulso, e $\|\mathbf{p}\|_{1}$ e $\|\mathbf{p}\|_{2}$ são, respectivamente, a norma-1 e norma-2 de p. A medida $S(\mathbf{p})$ varia de 0 (grau de esparsidade do filtro uniforme) a 1 (esparsidade do filtro de Dirac). Utilizando (12), o grau de esparsidade da resposta ao impulso apresentado acima é $S(\mathbf{p})=0,9435$. O sinal de entrada é correlacionado, com média zero e variância unitária, obtido através de um processo AR(2) dado por

$$
x(n)=0,4 x(n-1)-0,4 x(n-2)+v(n)
$$

onde $v(n)$ é um ruído branco com variância $\sigma_{v}^{2}=0,77$. A dispersão dos autovalores da matriz de autocorrelação do vetor de entrada é $\chi=10$ e o ruído de medição $z(n)$ é branco com variância $\quad \sigma_{z}^{2}=10^{-3} \quad(\mathrm{SNR}=30 \mathrm{~dB})$. Para avaliar $\quad 0$ desempenho do algoritmo, utiliza-se o desalinhamento normalizado (em $\mathrm{dB})$, dado por [3]

$$
\kappa(n)=10 \log _{10} \frac{\|\mathbf{p}-\mathbf{w}(n)\|_{2}^{2}}{\|\mathbf{p}\|_{2}^{2}} .
$$

Para mostrar a dependência do algoritmo PNLMS em relação ao fator de ativação, algumas curvas de desalinhamento são mostradas na Fig. 1, considerando $f(n)$ dado por (6), $\mu=0,5, \delta=0,01$ e usando $\rho$ igual a 0,01, 0,05 e 0,50. Além disso, uma quarta simulação numérica, agora usando um fator de ativação constante $f(n)=0,001$, manualmente ajustado para maximizar a velocidade de convergência, é também incluída na Fig. 1. Essa figura mostra que a convergência do algoritmo é impactada pelo fator de ativação bem como pelo parâmetro $\rho$. Note que, usando $f(n)$ dado em (6) e reduzindo o valor de $\rho$, a velocidade de convergência aumenta. Para o caso em que $f(n)=0,001$, uma convergência mais rápida é obtida, visto que o algoritmo comporta-se de maneira mais proporcional, isto é, um menor ganho por iteração é atribuído aos coeficientes inativos. Dessa forma, a partir das curvas mostradas na Fig. 1, um fato interessante pode ser inferido: o fator de ativação dado por (6) não é o melhor caminho para se obter uma distribuição de ganhos adequada.

Para avaliar a distribuição de ganhos do algoritmo, as seguintes figuras de mérito são definidas:

Distribuição de ganho total em $L$ iterações

$$
\theta_{i}=\sum_{n=0}^{L-1} g_{i}(n)
$$

Média dos ganhos totais $\theta_{i}$ atribuídos aos coeficientes inativos

$$
\theta_{\text {med }}^{\text {inativo }}=\frac{1}{N-N_{\text {ativo }}} \sum_{i \notin A} \theta_{i} .
$$

A Tabela I resume as expressões (15) e (16) para os casos considerados na Fig. 1, usando $N_{\text {ativo }}=4$ e $A=\{1,30,35$,

85\}. Note na tabela que, no caso do fator de ativação constante $[f(n)=0,001]$, uma maior parcela de ganho dos coeficientes inativos é transferida para os ativos, resultando em uma melhor distribuição de ganhos de adaptação.

TABELA I

DISTRIBUIÇÃO DE GANHOS TOTAIS DO ALGORITMO PNLMS EM 5000 ITERAÇÕES

\begin{tabular}{c|c|c|c|c|c}
\hline \hline Parâmetros & $\theta_{1}$ & $\theta_{30}$ & $\theta_{35}$ & $\theta_{85}$ & $\theta_{\text {med }}^{\text {inativo }}$ \\
\hline$\rho=0,01$ & 198,0 & 1868,5 & 924,5 & 176,8 & 19,1 \\
\hline$\rho=0,05$ & 80,5 & 765,3 & 376,2 & 72,1 & 38,6 \\
\hline$\rho=0,50$ & 49,9 & 98,6 & 49,5 & 49,5 & 49,5 \\
\hline$f(n)=0,001$ & 283,9 & 2749,3 & 1364,0 & 262,2 & 3,5 \\
\hline \hline
\end{tabular}

A Fig. 2 mostra o comportamento inicial dos coeficientes ativos $w_{1}(n), w_{30}(n)$ e $w_{35}(n)$, do coeficiente inativo $w_{2}(n)$ e dos ganhos $g_{1}(n), g_{2}(n), g_{30}(n)$ e $g_{35}(n)$. Analisando a Fig. 2(a) no início da fase de aprendizagem $(0 \leq n<30)$, note que $\left|w_{30}(n)\right|<\left|w_{1}(n)\right|$, resultando em $g_{30}(n)<g_{1}(n) \quad$ [veja Fig. 2(b)]. Esse comportamento não é desejável, visto que $g_{1}(n)$ e $g_{30}(n)$ não são proporcionais às magnitudes dos correspondentes coeficientes da planta $p_{1}$ e $p_{30}$, respectivamente, onde $\left|p_{1}\right|=0,1$ e $\left|p_{30}\right|=1,0$, em contraste à condição desejada $g_{30}(n)>g_{1}(n)$. Esse fato assinala que o algoritmo PNLMS pode apresentar um comportamento inadequado no início do processo de adaptação. Tal problema é considerado na próxima seção para a formulação de uma versão melhorada do algoritmo PNLMS.

\section{AlgORITMO PNLMS MODIFICADO}

Considerando a análise anterior do algoritmo PNLMS, agora concentramos a nossa discussão nas seguintes características:

i) Quando $w_{i}(n)$ é um coeficiente ativo, note de (10) que seu ganho é sempre proporcional a $\left|w_{i}(n)\right|$.

ii) Quando $w_{i}(n)$ é inativo, observe de (9) que o ganho não é proporcional a $\left|w_{i}(n)\right|$. 


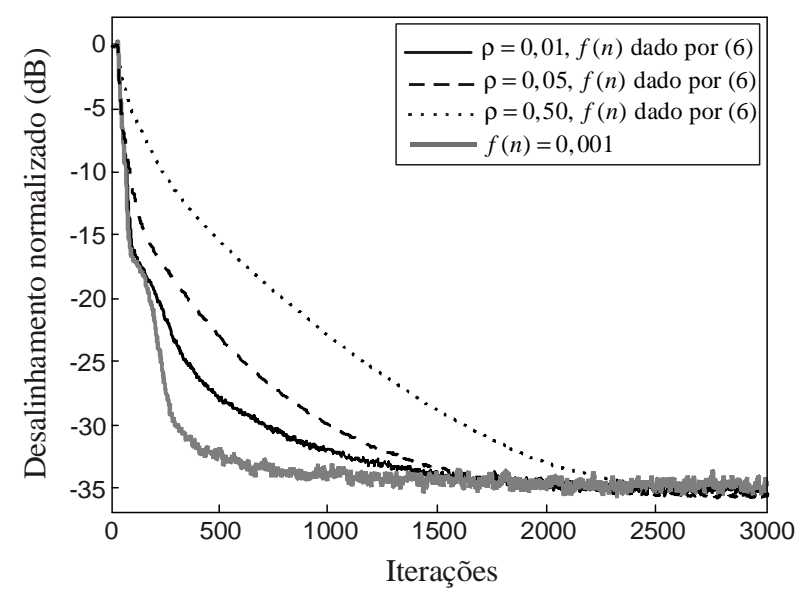

Fig. 1. Desalinhamento normalizado do algoritmo PNLMS usando $\mu=0,5$ e $\delta=0,01$.

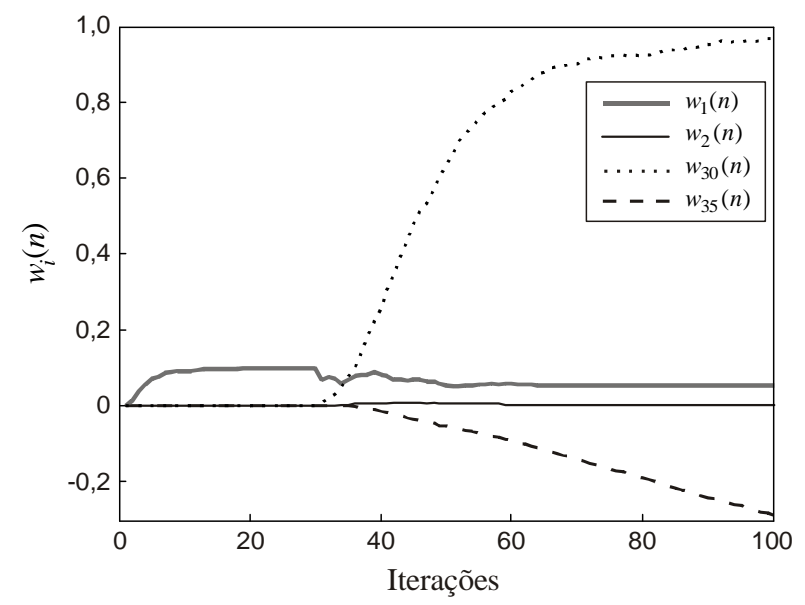

(a)

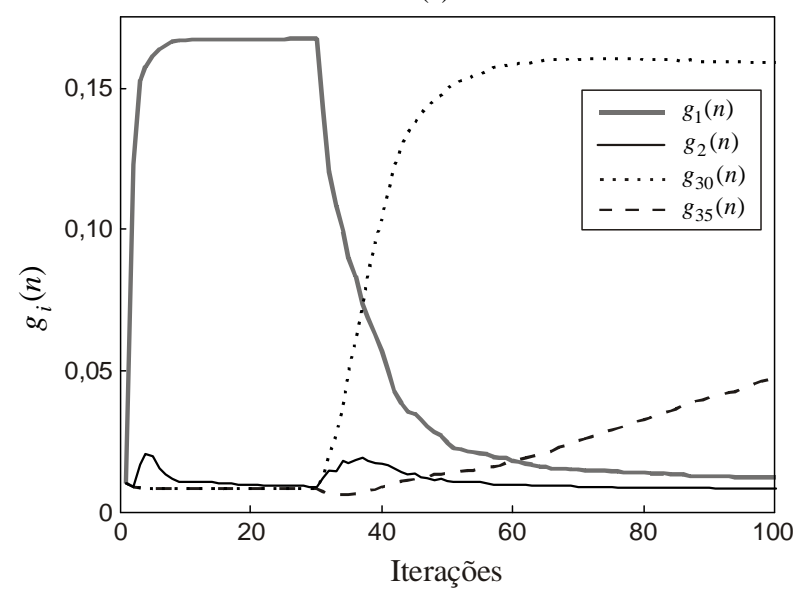

(b)

Fig. 2. Evolução das variáveis do algoritmo PNLMS com $\mu=0,5, \rho=0,05$ e $\delta=0,01$. (a) Coeficientes $w_{1}(n), w_{2}(n), w_{30}(n)$ e $w_{35}(n)$. (b) Ganhos $g_{1}(n), g_{2}(n), g_{30}(n)$ e $g_{35}(n)$.

A característica (i) é desejável visto que a mesma está de acordo com a filosofia proporcional. Por outro lado, (ii) não está em concordância com o conceito de proporcionalidade. Aqui, o objetivo é contornar tal desvantagem fazendo o ganho $g_{i}(n)$ tender a ser proporcional a $\left|w_{i}(n)\right|$ mesmo que $w_{i}(n)$ seja inativo.
Com o propósito de se obter um algoritmo completamente proporcional, associa-se cada ganho atribuído a um coeficiente inativo com um fator de ativação individual $f_{i}(n)$, em lugar de um fator comum para todos os coeficientes inativos. Assim, substituindo $f(n)$ por $f_{i}(n)$ em (5), a função de proporcionalidade é reescrita como

$$
\phi_{i}(n)=\max \left[f_{i}(n),\left|w_{i}(n)\right|\right]
$$

e, em conseqüência, quando $w_{i}(n)$ é inativo, a partir de (4) e (17), (9) é modificada para

$$
g_{i}^{\text {inativo }}(n)=\frac{1}{c(n)} f_{i}(n) .
$$

\section{A. Condições Requeridas para o Novo Fator de Ativação}

Agora, as condições requeridas para cada fator de ativação individual $f_{i}(n)$ são estabelecidas como segue:

C1) $f_{i}(n)$ deve convergir para a correspondente magnitude do coeficiente $\left|w_{i}(n)\right|$, isto é,

$$
\lim _{n \rightarrow \infty}\left[f_{i}(n)-\left|w_{i}(n)\right|\right]=0, \quad i=1,2, \ldots, N .
$$

C2) $f_{i}(n)$ deve sempre ser maior do que zero, isto é,

$$
f_{i}(n)>0, \quad i=1,2, \ldots, N .
$$

Assim, se C1 for satisfeita, note a partir de (18) que $g_{i}^{\text {inativo }}(n)$ tende a ser proporcional a $\left|w_{i}(n)\right|$ quando $n \rightarrow \infty$. Além disso, a condição C2 garante que $g_{i}(n)>0$ quando $\left|w_{i}(n)\right|=0$, evitando o congelamento de $w_{i}(n)$.

\section{B. Abordagem Proposta para Determinar $f_{i}(n)$}

Nesta seção, o objetivo é fazer o fator de ativação individual $f_{i}(n)$ tender à magnitude $\left|w_{i}(n)\right|$. Note que, no início do processo de adaptação $(n=1)$, os únicos dados disponíveis são a estimativa do i-ésimo coeficiente $w_{i}(1)$ e a função de proporcionalidade $\phi_{i}(0)$. De (17), verifica-se que $\phi_{i}(0)=f_{i}(0)$, visto que o filtro adaptativo é inicializado com $\mathbf{w}(0)=\mathbf{0}$. Então, é razoável a seguinte formulação para calcular o fator de ativação $f_{i}(1)$ :

$$
f_{i}(1)=\gamma_{i}\left|w_{i}(1)\right|+\left(1-\gamma_{i}\right) \phi_{i}(0)
$$

onde $0<\gamma_{i}<1$. Aqui, os fatores de ativação são inicializados usando um pequeno valor constante positivo (tipicamente, $\left.f_{i}(0)=10^{-2} / N\right)$, tal que $f_{i}(0)>0$. Em (19), o primeiro termo do segundo membro é devido ao objetivo pretendido e o segundo, garante que $f_{i}(1)$ seja sempre maior do que zero. Agora, generalizando essa abordagem para todo $n$, obtém-se

$$
f_{i}(n)=\gamma_{i}\left|w_{i}(n)\right|+\left(1-\gamma_{i}\right) \phi_{i}(n-1) .
$$

Considerando que não se tem conhecimento a priori da planta (posição dos coeficientes ativos, tipo de perturbação a que a planta será submetida), é razoável escolher $\gamma_{i}=1 / 2$, dessa 
forma, ponderando igualmente $\left|w_{i}(n)\right|$ e $\phi_{i}(n-1)$. Assim, obtém-se

$$
f_{i}(n)=\frac{1}{2}\left|w_{i}(n)\right|+\frac{1}{2} \phi_{i}(n-1)
$$

Por recursão, usando (17) e (21) a partir do instante 1 até $n$, pode-se mostrar que $f_{i}(n)$, dado por (21), satisfaz simultaneamente as condições C1 e C2. Note de (21) que agora $f_{i}(n)$ depende de $\left|w_{i}(n)\right|$. Então, para uma operação apropriada do algoritmo, requer-se que as magnitudes instantâneas dos coeficientes estimados sejam proporcionais às magnitudes dos correspondentes coeficientes da planta. Contudo, $\left|w_{i}(n)\right|$ pode não ser proporcional a $\left|p_{i}(n)\right|$ no início do processo de adaptação [veja Fig. 2(a)], o que pode ser contornado fazendo-se a atualização periódica de $f_{i}(n)$ somente após um período de aprendizagem de $N$ amostras (igual ao comprimento do filtro adaptativo). Portanto, (21) é reescrita como

$$
f_{i}(n)= \begin{cases}\frac{1}{2}\left|w_{i}(n)\right|+\frac{1}{2} \phi_{i}(n-1), & n=m N, \quad m=1,2,3, \ldots \\ f_{i}(n-1), & \text { caso contrário } .\end{cases}
$$

As expressões (17) e (22) caracterizam o algoritmo IAF-PNLMS proposto. Agora, cada coeficiente, seja ativo ou inativo, tem um fator de ativação associado $f_{i}(n)$ calculado por (22).

\section{Complexidade Computacional e Memória Requerida}

$\mathrm{O}$ algoritmo proposto requer uma memória adicional de tamanho $2 N$ para armazenar ambos $\phi_{i}(n-1)$ e $f_{i}(n-1)$. Por outro lado, o cálculo de (6) (exigido pelo algoritmo PNLMS) não é mais necessário no algoritmo IAF-PNLMS, economizando-se $N$ operações de comparação e uma operação de multiplicação. No que concerne à determinação de (22), $N$ adições e $N$ multiplicações são requeridas a cada $N$ amostras, resultando em uma adição e uma multiplicação por iteração.

\section{Resultados de Simulação}

Nesta seção, simulações de Monte Carlo (média de 100 realizações independentes) dos algoritmos NLMS, PNLMS, IPNLMS e IAF-PNLMS são realizadas, objetivando comparar velocidade de convergência e resposta a perturbações na planta, considerando um problema de identificação de sistema. Além disso, a distribuição de ganhos do algoritmo IAF-PNLMS é avaliada. Para tal, três exemplos são apresentados, utilizando-se o mesmo cenário de simulação especificado na Seção II. B.

\section{A. Exemplo 1}

Neste exemplo, os algoritmos NLMS, PNLMS, IPNLMS e IAF-PNLMS são comparados em termos da velocidade de convergência e da resposta a uma perturbação na planta, considerando que o vetor de coeficientes da planta $\mathbf{p}$ muda para $-\mathbf{p}$ no instante $n=2500$.
A Fig. 3(a) ilustra as curvas de desalinhamento normalizado dos algoritmos NLMS, PNLMS (com $\rho=0,05$ e $\delta=0,01$ ), IPNLMS e IAF-PNLMS [com $f_{i}(0)=10^{-4}$ ]. No algoritmo IPNLMS, $\alpha=0$ (um parâmetro desse algoritmo) é usado [6], [7]. Comparando as curvas na Fig. 3(a), observa-se que o algoritmo IAF-PNLMS atinge a mais rápida velocidade de convergência.

\section{B. Exemplo 2}

Aqui, novamente os algoritmos NLMS, PNLMS, IPNLMS e IAF-PNLMS são comparados em termos da velocidade de convergência e da resposta a uma perturbação na planta. Os mesmos valores dos parâmetros do Exemplo 1 são considerados. Agora, no instante $n=2500$, o vetor de coeficientes da planta p é deslocado de 12 amostras para a direita, alterando-se a posição de todos os coeficientes ativos. Dessa maneira, os valores dos coeficientes ativos da planta iguais a $\{0,1,1,0,-0,5,0,1\}$, localizados nas posições $\{1,30$, 35 , 85\}, são movidos para as posições $\{13,42,47,97\}$ após $n=2500$.

A Fig. 3(b) mostra as curvas de desalinhamento normalizado dos algoritmos NLMS, PNLMS (com $\rho=0,05$ e $\delta=0,01$ ), IPNLMS (com $\alpha=0$ ) e IAF-PNLMS [com $f_{i}(0)=10^{-4}$ ]. Note nessa figura que o algoritmo IAF-PNLMS novamente atinge a mais rápida velocidade de convergência.

\section{Exemplo 3}

Neste exemplo, a distribuição de ganhos totais em 5000 iterações do algoritmo IAF-PNLMS é avaliada. As mesmas condições e perturbação na planta do Exemplo 1 são utilizadas. Além disso, para avaliar a distribuição de ganhos totais, define-se o fator de ativação ideal $f_{i}^{\text {ideal }}(n)$ como

$$
f_{i}^{\text {ideal }}(n)=\left|p_{i}\right|
$$

onde $\left|p_{i}\right|$ é a magnitude do i-ésimo coeficiente da planta. Essa definição é razoável, visto que (assumindo a convergência do algoritmo) $f_{i}(\infty) \rightarrow\left|w_{i}(\infty)\right|$ satisfaz a condição C1. Assim, para o cenário de simulação deste exemplo, (23) resulta em

$$
f_{i}^{\text {ideal }}(n)= \begin{cases}0,1, & i=1,85 \\ 1,0, & i=30 \\ 0,5, & i=35 \\ 0,0, & \text { caso contrário. }\end{cases}
$$

A Tabela II apresenta a distribuição de ganhos totais do algoritmo IAF-PNLMS (com $L=5000$ iterações) usando $f_{i}(0)=10^{-4}$. Além disso, nessa tabela, uma segunda distribuição de ganhos totais do algoritmo IAF-PNLMS usando $f_{i}^{\text {ideal }}(n)$ é mostrada. Note que as distribuições de ganhos obtidas para ambos os casos são numericamente muito similares, indicando que a regra proposta em (22), usada para se obter $f_{i}(n)$, é razoável. Aqui, é importante salientar que o uso do fator de ativação ideal não é uma solução prática, visto que em geral $\left|p_{i}\right|$ não é conhecido a priori. $\mathrm{O}$ fator de ativação ideal foi introduzido somente para propósito de comparação. 


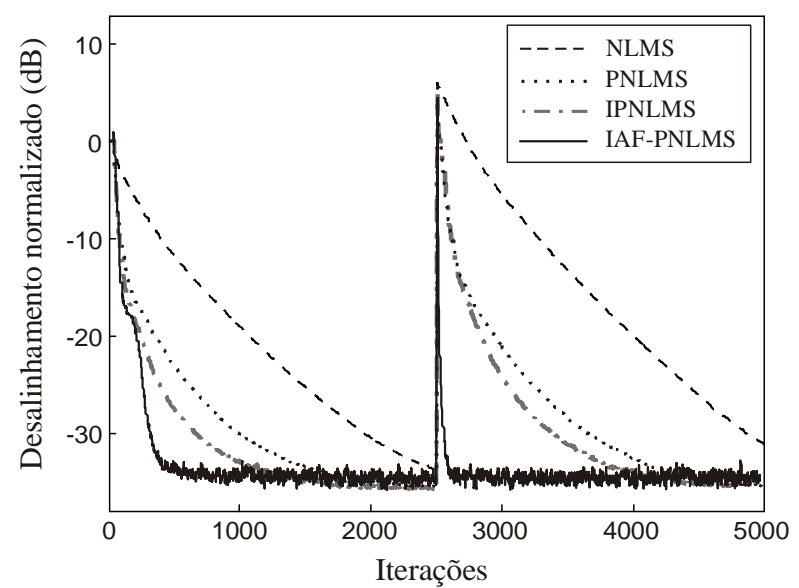

(a)

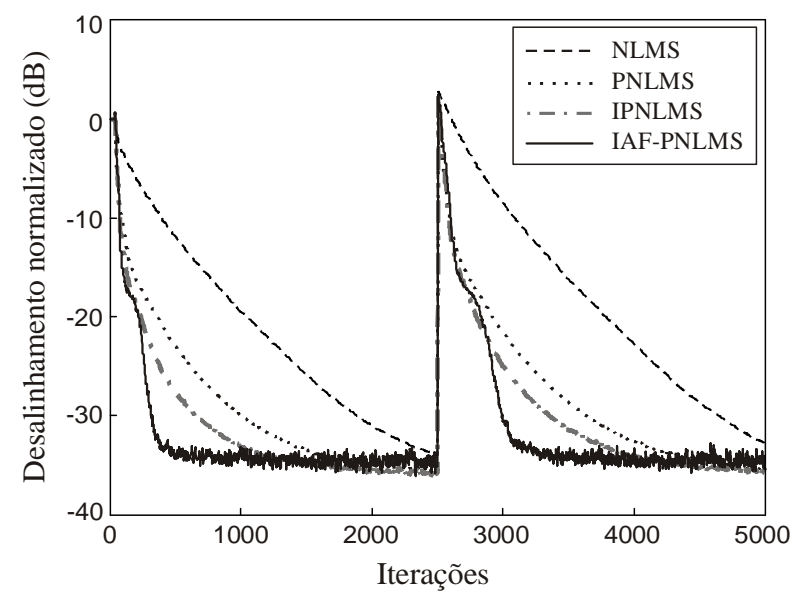

(b)

Fig. 3. Curvas de desalinhamento normalizado usando $\mu=0,5, \quad \rho=0,05$, $\delta=0,01, \quad f_{i}(0)=10^{-4}$ e $\alpha=0$. (a) Exemplo 1: sinal dos coeficientes da planta é invertido em $n=2500$. (b) Exemplo 2: deslocamento dos coeficientes da planta em $n=2500$.

TABELA II

EXEMPLO 3. DISTRIBUIÇÃO DE GANHOS TOTAIS DO ALGORITMO IAF-PNLMS EM 5000 ITERAÇÕES

\begin{tabular}{c|c|c|c|c|c}
\hline \hline $\begin{array}{c}\text { Fatores de } \\
\text { Ativação }\end{array}$ & $\theta_{1}$ & $\theta_{30}$ & $\theta_{35}$ & $\theta_{85}$ & $\theta_{\text {med }}^{\text {inativo }}$ \\
\hline$f_{i}(0)=10^{-4}$ & 318,2 & 2860,8 & 1430,4 & 282,4 & 1,1 \\
\hline$f_{i}^{\text {ideal }}(n)$ & 300,0 & 2929,6 & 1470,8 & 299,6 & 0,0 \\
\hline \hline
\end{tabular}

\section{CONCLUSÕES}

Neste trabalho, uma nova versão melhorada do algoritmo PNLMS, denominada IAF-PNLMS, é proposta, considerando fatores de ativação individuais para cada coeficiente do filtro adaptativo. Cada fator de ativação é calculado em termos da magnitude de seu correspondente coeficiente e não mais depende dos parâmetros de proporcionalidade e inicialização considerados no algoritmo PNLMS. O algoritmo IAF-PNLMS apresenta uma melhor distribuição de ganhos do que os algoritmos PNLMS e IPNLMS, conduzindo a uma maior velocidade de convergência, superando os algoritmos PNLMS e IPNLMS para respostas ao impulso com grau de esparsidade elevado.

\section{REFERÊNCIAS}

[1] B. Jelfs, D. P. Mandic, and J. Benesty, "A class of adaptively regularised PNLMS algorithms,” in Proc. 15th Int. Conf. Digital Signal Process., Cardiff, UK, Jul. 2007, pp. 19-22.

[2] R. K. Martin, W. A. Sethares, R. C. Williamson, and C. R. Johnson, Jr., "Exploiting sparsity in adaptive filters," IEEE Trans. Signal Process., vol. 50, no. 8, pp. 1883-1894, Aug. 2002.

[3] Y. Huang, J. Benesty, and J. Chen, Acoustic MIMO Signal Processing. New York: Springer-Verlag, 2006.

[4] D. L. Duttweiler, "Proportionate normalized least-mean-squares adaptation in echo cancellers," IEEE Trans. Speech Audio Process., vol. 8, no. 5, pp. 508-518, Sep. 2000.

[5] S. L. Gay, “An efficient, fast converging adaptive filter for network echo cancellation" in Proc. Thirty-Second Asilomar Conf. Signals, Systems and Computers, Monterey, USA, Nov. 1998, vol. 1, pp. 394-398.

[6] J. Benesty and S. L. Gay, “An improved PNLMS algorithm," in Proc. IEEE Int. Conf. Acoust., Speech, Signal Process. (ICASSP), Orlando, USA, May 2002, vol. 2, pp. 1881-1884.

[7] J. Cui, P. A. Naylor, and D. T. Brown, "An improved IPNLMS algorithm for echo cancellation in packet-switched networks," in Proc. IEEE Int. Conf. Acoust., Speech, Signal Process. (ICASSP), Montreal, Canada, May 2004, vol. 4, pp. 141-144.

[8] S. L. Gay and S. C. Douglas, "Normalized natural gradient adaptive filtering for sparse and non-sparse systems," in Proc. IEEE Int. Conf. Acoust., Speech, Signal Process. (ICASSP), Orlando, USA, May 2002, vol. 2, pp. 1405-1408.

[9] S. Werner, J. A. Apolinário, P. S. R. Diniz, and T. I. Laakso, “A set-membership approach to normalized proportionate adaptation algorithms," in Proc. European Signal Process. Conf. (EUSIPCO), Antalya, Turkey, Sep. 2005, pp. 1-4.

[10] H. Deng and M. Doroslovacki, "Proportionate adaptive algorithms for network echo cancellation,” IEEE Trans. Signal Process., vol. 54, no. 5, pp. 1794-1803, May 2006.

[11] P. A. Naylor, J. Cui, and M. Brookes, “Adaptive algorithms for sparse echo cancellation,” Signal Process., vol. 86, no. 6, pp. 1182-1192, Jun. 2006.

[12] B. Jelfs, D. P. Mandic, and A. Cichocki, "A unifying approach to the derivation of the class of PNLMS algorithms," in Proc. 15th Int. Conf. Digital Signal Process., Cardiff, UK, Jul. 2007, pp. 35-38.

[13] J. Benesty and Y. Huang, "The LMS, PNLMS, and exponentiated gradient algorithms," in Proc. European Signal Process. Conf. (EUSIPCO), Vienna, Austria, Sep. 2004, pp. 721-724.

[14] P. Loganathan, A. W. H. Khong, and P. A. Naylor, "A sparseness controlled proportionate algorithm for acoustic echo cancellation,” in Proc. European Signal Process. Conf. (EUSIPCO), Lausanne, Switzerland, Aug. 2008, pp. 1-5.

[15] P. O. Hoyer, "Non-negative matrix factorization with sparseness constraints,” J. Mach. Learning Research, vol. 5, pp. 1457-1469, Nov. 2004. 